\title{
In vitro characterization and in vivo evaluation of nanostructured lipid curcumin carriers for intragastric administration
}

This article was published in the following Dove Press journal:

International Journal of Nanomedicine

8 October 2012

Number of times this article has been viewed

\section{Min Fang \\ Yilin Jin \\ Wei Bao \\ Hui Gao \\ Mengjin Xu \\ Di Wang \\ Xia Wang \\ Ping Yao \\ Liegang Liu}

Department of Nutrition and Food Hygiene, Hubei Key Laboratory of Food Nutrition and Safety, and Ministry of Education Key Laboratory of Environment and Health, School of Public Health, Tongji Medical College, Huazhong University of Science and Technology, I 3 Hangkong Road, Wuhan, People's Republic of China
Correspondence: Liegang Liu Department of Nutrition and Food Hygiene and MOE Key Laboratory of Environment and Health, School of Public Health, Tongji Medical College, Huazhong University of Science and Technology, 13 Hangkong Road, Wuhan 430030,

People's Republic of China

Tel +862783650522

$\mathrm{Fax}+862783650522$

Email Igliu@mails.tjmu.edu.cn
Background: Curcumin has a variety of pharmacological effects. However, poor water solubility and low oral bioavailability limit its clinical utility. A delivery system for nanostructured lipid carriers has been reported to be a promising approach to enhancing the oral absorption of curcumin. The aim of the present study was to investigate the pharmacokinetics, tissue distribution, and relative bioavailability of curcumin in rats after a single intragastric dose of a nanostructured lipid curcumin carrier formulation.

Methods: Nanostructured lipid curcumin carriers were prepared using the ethanol dripping method and characterized in terms of the particle size, polydispersity index, zeta potential, differential scanning calorimetry, drug-loading capacity, encapsulation efficiency, and in vitro release. The pharmacokinetics and tissue distribution of nanostructured lipid curcumin carriers and curcumin suspension were compared after intragastric administration.

Results: Nanostructured lipid curcumin carriers showed a significantly higher peak plasma concentration (564.94 $\pm 14.98 \mathrm{ng} / \mathrm{mL}$ versus $279.43 \pm 7.21 \mathrm{ng} / \mathrm{mL}, P<0.01$ ), a shorter time taken to reach peak plasma concentration $(0.5 \pm 0.01$ hour versus $1.0 \pm 0.12$ hour, $P<0.01)$, and a greater $\mathrm{AUC}_{0-\infty}(820.36 \pm 25.11 \mathrm{mg} \times$ hour $/ \mathrm{L}$ versus $344.11 \pm 10.01 \mathrm{mg} \times$ hour $/ \mathrm{L}, P<0.05)$ compared with curcumin suspension. In the tissue distribution studies, curcumin could be detected in the spleen, heart, liver, kidneys, lungs, and brain. Following intragastric administration of the nanostructured lipid curcumin carrier formulation, tissue concentrations of curcumin also increased, especially in the brain. The nanostructured lipid curcumin carrier formulation improved the ability of curcumin to cross the blood-brain barrier, with an 11.93-fold increase in the area under the curve achieved in the brain when compared with curcumin suspension.

Conclusion: The nanostructured lipid carrier formulation significantly improved the oral bioavailability of curcumin and represents a promising method for its oral delivery.

Keywords: curcumin, oral bioavailability, tissue distribution

\section{Introduction}

Curcumin, a yellow-colored phenolic substance derived from the rhizome of the spice herb Curcuma longa, widely known as turmeric, has a broad spectrum of biological and pharmacological activity. Clinical trials have shown that curcumin has antioxidant, ${ }^{1-3}$ antiinflammatory, ${ }^{4,5}$ antibacterial, ${ }^{6}$ antifungal, ${ }^{7-9}$ and anticarcinogenic activity. ${ }^{10-14}$ Further, the cardioprotective and neuroprotective effects of curcumin are also well documented. ${ }^{15-17}$ The most compelling and key rationale for the therapeutic use of curcumin is its good safety profile. To date, no studies in either animals or humans have demonstrated any toxicity associated with the use of curcumin, even at high doses. ${ }^{18,19}$ Unfortunately, the potential use of curcumin is severely limited by its poor water solubility 
and short biological half-life, which results in low bioavailability irrespective of the route of administration..$^{20-22}$

Several approaches have been investigated to increase the oral bioavailability of curcumin, including nanoparticles, liposomes, micelles, and phospholipid complexes. ${ }^{20}$ Solid lipid nanoparticles have received particular attention because they consist of biocompatible lipids with high drug encapsulation efficiency (EE), enhanced intestinal permeability, and good oral bioavailability. ${ }^{23}$ However, solid lipid curcumin nanoparticles offered no improvements over standard curcumin when tested in vitro. ${ }^{24}$ Nanostructured lipid carriers are the second generation of solid lipid nanoparticles and are composed of a binary mixture of solid lipids and a spatially different liquid lipid as a carrier. ${ }^{25}$ Nanostructured lipid carriers are a novel lipid formulation that offers the advantages of improved drug-loading capacity (LC) and release properties. Furthermore, nanostructured lipid carriers can be prepared for multiple routes of administration, including oral, intravenous, pulmonary, and transdermal formulations. ${ }^{25}$ To date, intragastric administration of a nanostructured curcumin lipid carrier (CUR-NLC) formulation has not been described.

Recently, we prepared CUR-NLCs by an ethanol dripping method. The aim of this study was to describe the pharmacokinetics, tissue distribution, and relative bioavailability of curcumin in rats after single-dose intragastric administration of a CUR-NLC formulation in comparison with curcumin suspension.

\section{Materials and methods}

\section{Chemicals and reagent}

Standard curcumin and emodin (as the internal standard) were purchased from Fluka (Sigma-Aldrich, Buchs, Switzerland). Poloxamer 188 was purchased from Chemie (Sigma-Aldrich Chemie, Munich, Germany) and glyceryl monostearate was purchased from Tokyo Chemical Industry Co, Ltd (Tokyo, Japan). Soy lecithin was purchased from Shanghai Taiwei Medical Group Co, Ltd (Shanghai, China). Medium chain triglycerides $\left(\mathrm{Lexol}^{\circledR}\right)$ were sourced from Zhejiang WuMei Chemical Products Co, Ltd (Zhejiang, China). Methanol and acetonitrile (chromatographic grade) were obtained from Fisher Scientific Inc (Waltham, MA). Water for highperformance liquid chromatography (HPLC) was doubledistilled. All other chemicals and reagents were of analytical grade and were used without further purification.

\section{Animals}

Healthy Sprague-Dawley rats weighing 190-220 g were purchased from the Animal Institution, Zhongnan Hospital,
Wuhan University. The rats were housed under standard conditions, and provided with fresh water and a commercial diet ad libitum. The animal tests were performed in accordance with the Guide for the Care and Use of Laboratory Animals of the National Research Council. All animal experiments were approved by the local animal ethics committee.

\section{Preparation of CUR-NLCs}

Nanostructured lipid carriers, empty or loaded with curcumin, were prepared using an ethanol dripping method. ${ }^{26}$ Briefly, an aqueous surfactant solution consisting of $1 \%(\mathrm{w} / \mathrm{w})$ Poloxamer 188 was prepared and heated to $75^{\circ} \mathrm{C}$ prior to addition of the lipid phase. To prepare the lipid phase, the selected solid and liquid lipid mixture was heated to $5^{\circ} \mathrm{C}-10^{\circ} \mathrm{C}$ above its melting point. An ethanolic solution of soy lecithin $(0.64 \mathrm{mmol})$ was added under stirring to the melted lipid $(1.18 \mathrm{mmol})$. For obtaining drug-loaded nanoparticles, curcumin $(0.32 \mathrm{mmol})$ was added under mechanical stirring. To obtain nanostructured lipid carriers, the ethanol solution containing lipid, lecithin, and curcumin was dripped into an aqueous surfactant solution with high-speed homogenization (A200-18G-S; Shanghai Angyi Instruments, Shanghai, China) at 10,000 rpm for 15 minutes. The ratio of the ethanolic lipid phase to the aqueous phase was 1:3(v/v) in the final suspension. The suspension was then dispersed in cold twice-distilled water $\left(100 \mathrm{~mL}\right.$ at $\left.2^{\circ} \mathrm{C}-3^{\circ} \mathrm{C}\right)$ containing $5 \%(\mathrm{w} / \mathrm{w})$ sucrose under continuous magnetic stirring at $1000 \mathrm{rpm}$ for 30 minutes. Successively, the nanostructured lipid carriers were submitted to exhaustive dialysis using a Visking 18/32 membrane (molecular cutoff 12,000-14,000 $\mathrm{Da}$ ), freeze-dried by a lyophilizer (Alpha 1-4; Martin Christ, Osterode am Harz, Germany) and stored at $-20^{\circ} \mathrm{C}$ in a freezer. The dry powder was kept at room temperature for successive characterization. Ethanol was completely removed from the nanoparticles during purification.

\section{Characterization of nanostructured lipid carriers}

The mean particle size, polydispersity index, and zeta potential of the nanostructured lipid carriers were determined by dynamic light scattering using a nanoparticle analysis instrument (Delsa ${ }^{\mathrm{TM}}$; Beckman Coulter, Fullerton, CA). Morphological examination of the nanostructured lipid carriers was performed using a transmission electron microscope (Hitachi Ltd, Tokyo, Japan).

Differential scanning calorimetry experiments can be used to determine thermodynamic variations related to 
morphological changes as a result of melting points and melting enthalpies varying with lipid modification. In the present study, crystalline state evaluation of blank nanostructured lipid carriers, curcumin, CUR-NLCs, and a physical mixture of blank nanostructured lipid carriers and curcumin (with same ratio as that of the CUR-NLCs) was conducted using a differential scanning calorimeter (Diamond; PerkinElmer Instruments, Boston, MA). A heating rate of $10^{\circ} \mathrm{C}$ per minute was used, and the temperature range was $50^{\circ} \mathrm{C}-220^{\circ} \mathrm{C}$. According to our previous work, no melting peak is detected in the range of $30^{\circ} \mathrm{C}-50^{\circ} \mathrm{C}$.

LC $\%$ and EE\% were determined by HPLC. ${ }^{27}$ Briefly, the CUR-NLC suspensions were first dissolved and diluted with anhydrous methanol. The suspensions were then centrifuged at 11,000 rpm for 15 minutes, after which the supernatant was determined by the HPLC method and the total amount of curcumin in the lipid nanoparticles was calculated. To determine the amount of curcumin contained in the nanostructured lipid carriers, equal volumes of the CUR-NLC formulation were accurately dispensed into a $0.9 \% \mathrm{NaCl}$ solution at a ratio of $5: 1(\mathrm{v} / \mathrm{v})$ to salt out the nonincorporated drug, and the suspensions were centrifuged at 18,000 rpm for 30 minutes. The upper portions of the suspensions were then diluted with anhydrous methanol, sonicated, and centrifuged at 11,000 rpm for 15 minutes. Finally, the supernatant was analyzed under the same HPLC conditions. For sample analysis, chromatography was performed using a Waters Alliance HPLC 2695 series (Waters Technologies, Milford, MA) with separation on a Thermo Hypersil ODS C18 Column $(250 \mathrm{~mm} \times 4.6 \mathrm{~mm}$, $5 \mu \mathrm{m}$; Hypersil, Thermo Scientific, Waltham, MA). The mobile phase consisted of acetonitrile and water (58:42, $\mathrm{v} / \mathrm{v}$ ) at a flow rate of $1 \mathrm{~mL}$ per minute. The run time for the analysis was 10 minutes and the detection wavelength was set at $423 \mathrm{~nm}$. The sample injection volume was $20 \mu \mathrm{L}$ and the column temperature was maintained at $30^{\circ} \mathrm{C}$. The $\mathrm{EE} \%$ and LC\% of the CUR-NLCs were calculated as follows:

$\mathrm{EE} \%=$ Amount of Curcumin Encapsulated in Nanostructured Lipid Carriers/Total Amount of Curcumin in Lipid Nanoparticles $\times 100 \%$.

$\mathrm{LC} \%=$ Amount of Curcumin Encapsulated in Nanostructured Lipid Carriers/Total Amount of Nanostructured Lipid Carriers $\times 100 \%$.

\section{In vitro release study of DXMA-NLCs}

The dialysis membrane method was used to investigate the in vitro release of curcumin from the CUR-NLC formulation. First, $2 \mathrm{~mL}$ of CUR-NLC solution (equivalent to
$1.5 \mathrm{mg}$ of curcumin) was transferred into dialysis bags with a molecular cutoff of $3.5 \mathrm{kDa}$. The bags were suspended in $250 \mathrm{~mL}$ of $\mathrm{pH} 6.8$ phosphate buffer (containing ethanol 15\% $\mathrm{v} / \mathrm{v}$ ) maintained at $37^{\circ} \mathrm{C} \pm 0.5^{\circ} \mathrm{C}$ in a shaking water bath at $100 \mathrm{rpm}$. At designated time intervals, $1 \mathrm{~mL}$ samples of the dialysis medium were taken for measurement of curcumin by HPLC, and the same volume of fresh medium was then added. The release experiments were performed in triplicate.

\section{Pharmacokinetics and tissue distribution studies of CUR-NLCs}

Sprague-Dawley rats received a single intragastric dose of the CUR-NLC formulation or curcumin suspension containing an equivalent curcumin dose of $80 \mathrm{mg} / \mathrm{kg}$. The rats were sacrificed at predetermined time points $(15,30$, and 45 minutes, and at 1, 2, 4, 6, 8, 12, and 24 hours) after dosing (five mice were sacrificed at each time point). Blood samples were collected from the orbital plexus into heparin-treated $(10 \mu \mathrm{L}, 500 \mathrm{IU} / \mathrm{mL})$ tubes and immediately centrifuged at $3000 \mathrm{rpm}$ for 15 minutes at $4^{\circ} \mathrm{C}$. Tissue samples from the heart, lung, liver, spleen, kidney, and brain were collected, washed, weighed, and homogenized. All of the samples were stored at $-80^{\circ} \mathrm{C}$ until later analysis.

Curcumin concentrations in plasma and tissues were determined by HPLC assay. Plasma or tissue homogenates $(300 \mu \mathrm{L})$ were mixed with $10 \mu \mathrm{L}$ of emodin $(20 \mu \mathrm{g} / \mathrm{mL})$ as the internal standard. The mixture was vortexed with $3 \mathrm{~mL}$ of acetic ether and centrifuged at $4000 \mathrm{rpm}$ for 10 minutes to precipitate the proteins. The supernatant was collected into clean test tubes and evaporated under a stream of nitrogen at $45^{\circ} \mathrm{C}$. The residues were dissolved in $100 \mu \mathrm{L}$ of mobile phase, and $20 \mu \mathrm{L}$ aliquots were injected into the HPLC system as described above.

\section{Pharmacokinetics and statistical analysis}

Pharmacokinetic analysis was carried out using 3P97 pharmacokinetic software (Chinese Pharmacological Association, Beijing, China). The statistical significance of differences in pharmacokinetic parameters between the treatment groups was determined with the Student's $t$-test using the Statistical Package for Social Sciences version 12.0 (IBM Corporation, Armonk, NY). Statistical significance was defined to be $P<0.05$ or $P<0.01$.

\section{Results and discussion Preparation and characterization of CUR-NLCs}

CUR-NLCs were prepared successfully by the ethanol dripping method, and their properties are shown in 
Table I Properties of the CUR-NLCs

\begin{tabular}{llllll}
\hline Sample & Particle size $(\mathbf{n m})$ & PI & Zeta potential $(\mathbf{m V})$ & EE $(\%)$ & LC $(\%)$ \\
\hline CUR-NLCs & $129 \pm 15.5$ & $-27.8 \pm 5.9$ & $0.257 \pm 0.079$ & $95.98 \pm 0.63$ & $4.21 \pm 0.07$ \\
\hline
\end{tabular}

Notes: Mean \pm SD, $\mathrm{n}=3$.

Abbreviations: CUR-NLCs, curcumin nanostructured lipid carriers; PI, polydispersity index; EE, encapsulation efficiency of CUR-NLCs; LC, drug-loading capacity of CUR-NLCs.

Table 1. The mean particle size, polydispersity index, zeta potential, EE, and LC of CUR-NLCs were $129 \pm 15.5 \mathrm{~nm}$, $-27.8 \pm 5.9 \mathrm{mV}, 0.257 \pm 0.079,95.98 \% \pm 0.63 \%$, and $4.21 \% \pm 0.07 \%$, respectively. The morphology of the CUR-NLCs recorded by transmission electron microscopy was spherical (Figure 1) and the particle size $(<200 \mathrm{~nm}$ ) was similar to that determined by dynamic light scattering. The in vitro release study showed sustained release of curcumin from the CUR-NLC formulation compared with the free curcumin suspension (Figure 2). While more than $90 \%$ of the free curcumin was found in the release medium after approximately 24 hours, the CUR-NLC formulation released less than $10 \%$ of its curcumin content after 1 hour and more than $30 \%$ after 24 hours. Differential scanning calorimetry was used to investigate the melting and crystallization behavior of the lipid materials, ie, to detect whether these characteristics were changed by formulation as CUR-NLCs. As shown in Figure $3 \mathrm{~A}$, the melting process for the blank nanostructured lipid carriers took place with maximum peaks at $55.53^{\circ} \mathrm{C}$ and $132.54^{\circ} \mathrm{C}$, respectively. Similarly, drug-loaded nanostructured lipid carriers showed melting points at $56.19^{\circ} \mathrm{C}$ and $134.67^{\circ} \mathrm{C}$ (Figure 3B). In addition, the physical mixture showed melting points at $55.20^{\circ} \mathrm{C}$ and $136.62^{\circ} \mathrm{C}$ (Figure $3 \mathrm{C}$ ). Curcumin alone showed a peak at $177.89^{\circ} \mathrm{C}$, as seen in Figure 3D. The results were obtained after one day of storage. Compared with blank nanostructured lipid carriers, the drug-loaded nanostructured

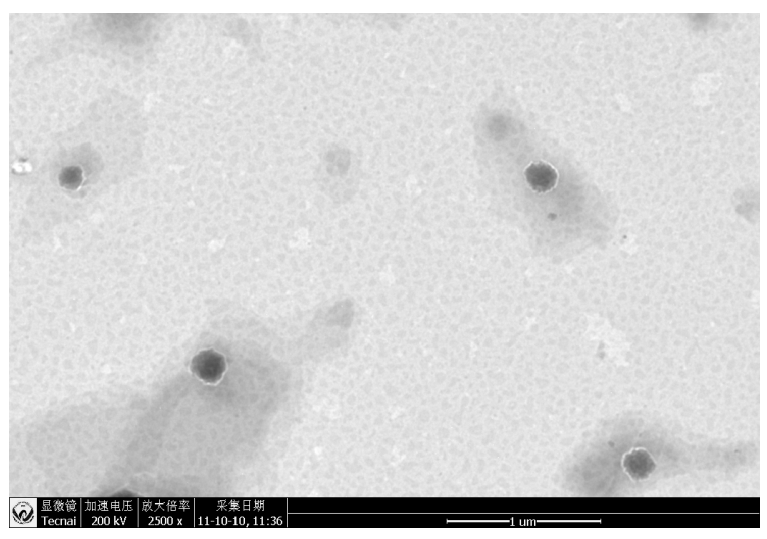

Figure I Transmission electron microscopy of CUR-NLCs. Note: Magnification: 2500x.

Abbreviation: CUR-NLCs, curcumin nanostructured lipid carriers. lipid carriers showed slower recrystallization and disturbance of crystalline order which might influence the loading capacity. There was no melting peak for curcumin observed in the differential scanning calorimetry thermograms for the physical mixture and the drug-loaded nanostructured lipid carriers. This absence may be explained by the low amount of curcumin $(4.21 \% \pm 0.07 \%)$ in the CUR-NLC formulation. From this result, it can be concluded that there was no important difference in this regard between the blank nanostructured lipid carriers, the drug-loaded nanostructured lipid carriers, and the physical mixture. A possible explanation may be that loading of a small amount of drug into the nanostructured lipid carriers did not change the melting point significantly. Similar results have been reported elsewhere. ${ }^{27,28}$

\section{Pharmacokinetic study and bioavailability}

Analytical methods for determination of curcumin in plasma are well established. ${ }^{29}$ The limit of quantification in plasma for curcumin measurement is $10 \mathrm{ng} / \mathrm{mL}$. The calibration curve over the range of $0.01-3.2 \mu \mathrm{g} / \mathrm{mL}$ for plasma had good linearity $\left(\mathrm{R}^{2}=0.9996\right)$. Intraday and interday accuracy precision was $2.7 \%$ and $3.3 \%$, respectively. Recovery of curcumin in plasma was $98.6 \%$.

The plasma concentration time profiles and corresponding pharmacokinetic parameters for curcumin and CUR-NLCs are shown in Figure 4 and Table 2. The pharmacokinetics

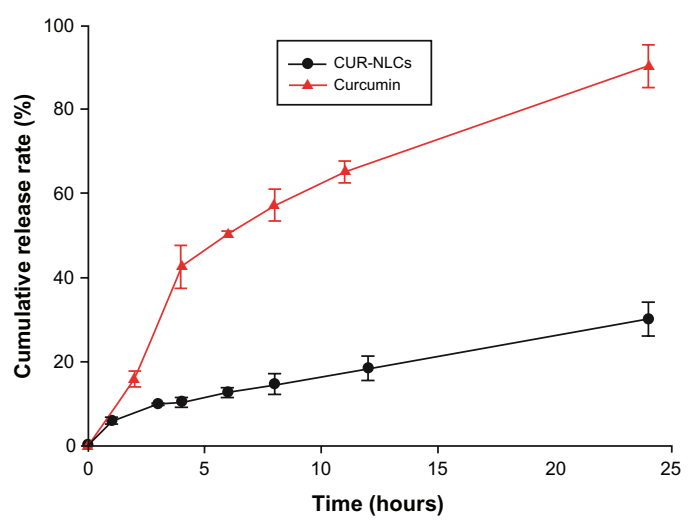

Figure 2 In vitro release profiles of curcumin from CUR-NLCs and curcumin suspension.

Notes: Mean \pm SD, $\mathrm{n}=3$.

Abbreviation: CUR-NLCs, curcumin nanostructured lipid carriers. 
of curcumin and CUR-NLCs fit a two-compartment model. As shown in Table 2, the pharmacokinetic parameters of the CUR-NLC solution in rats were markedly different from those of the curcumin suspension. The peak plasma concentration for CUR-NLCs was 2.02 times higher than the value for curcumin suspension. CUR-NLCs demonstrated a more rapid distribution phase $\left(\mathrm{t}_{1 / 2} \alpha=0.77 \pm 0.13\right.$ hours $)$ compared with curcumin suspension $\left(\mathrm{t}_{1 / 2} \alpha=1.19 \pm 0.27\right.$ hours $)$. In contrast, the elimination phase $\left(\mathrm{t}_{1 / 2} \beta\right)$ for the CUR-NLC formulation was longer ( $\mathrm{t}_{1 / 2} \beta=20.62 \pm 1.91$ hours $)$ compared with the curcumin suspension ( $\mathrm{t}_{1 / 2} \beta=6.47 \pm 0.95$ hours). The CURNLC formulation showed a higher $\mathrm{AUC}_{0-\infty}$ (2.38-fold) and mean residence time (2.32-fold) compared with the curcumin solution. Based on these data, the CUR-NLC formulation was found to improve the oral bioavailability of curcumin.
Curcumin was still measurable in rat plasma 24 hours after administration of the CUR-NLC formulation, but only up to 8 hours after administration of curcumin suspension. These results show that incorporation of curcumin into nanostructured lipid carriers resulted in increased absorption of curcumin on intragastric administration. It has been reported that the physicochemical environment of the gastrointestinal tract and intestinal epithelium acts as a barrier to curcumin absorption following oral administration. ${ }^{30,31}$ The nanostructured lipid carrier-modified curcumin formulation may enable bioadhesion at the wall of the gastrointestinal tract, thereby promoting absorption by increasing the duration of contact with the gastrointestinal surface. Furthermore, it has been reported that nanostructured lipid carriers are stable in the gastric environment, ${ }^{32,33}$ and after adhesion to the gut wall,

A

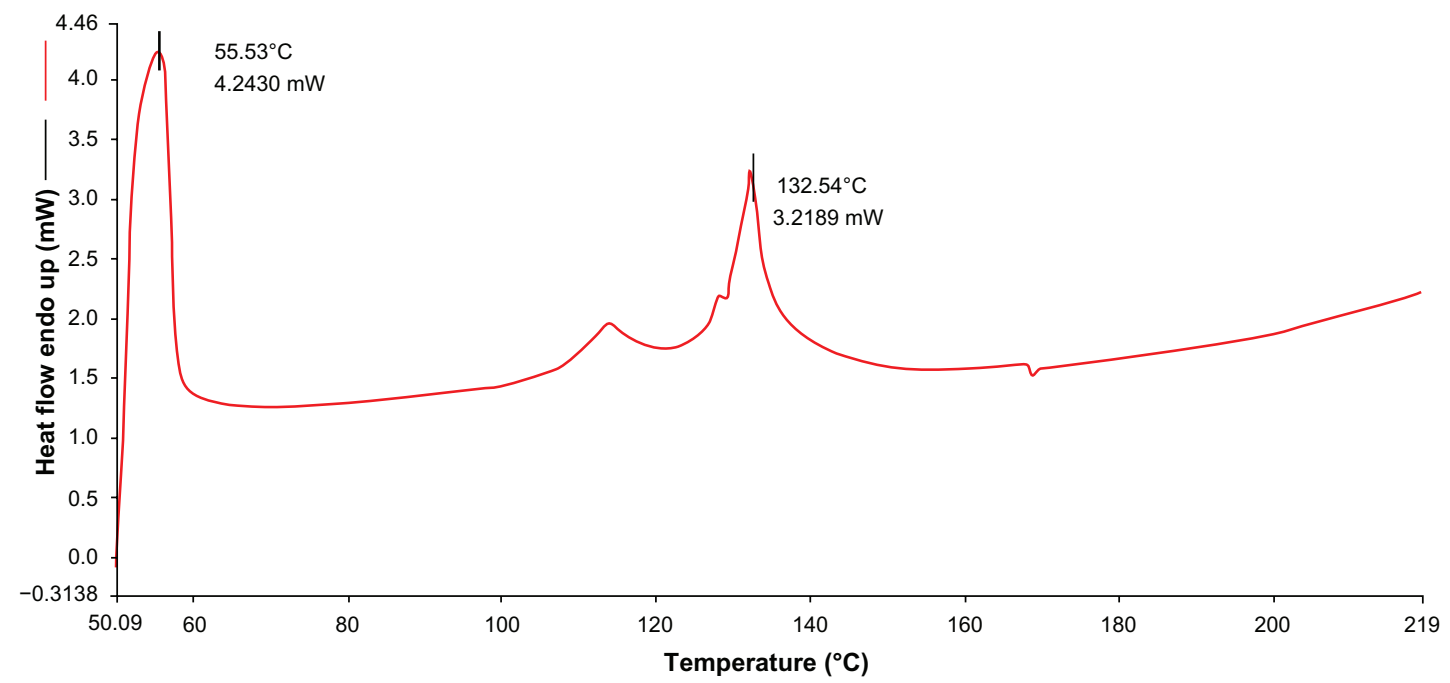

B

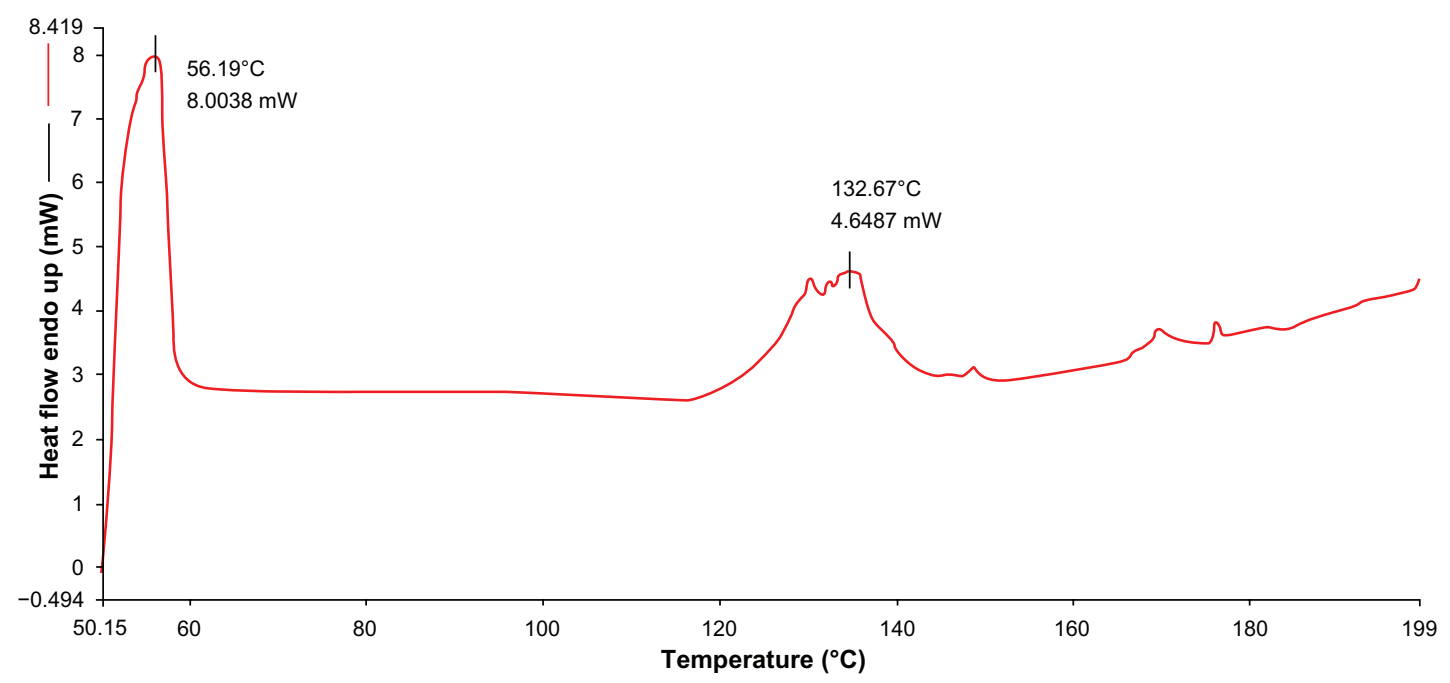

Figure 3 (Continued) 
C

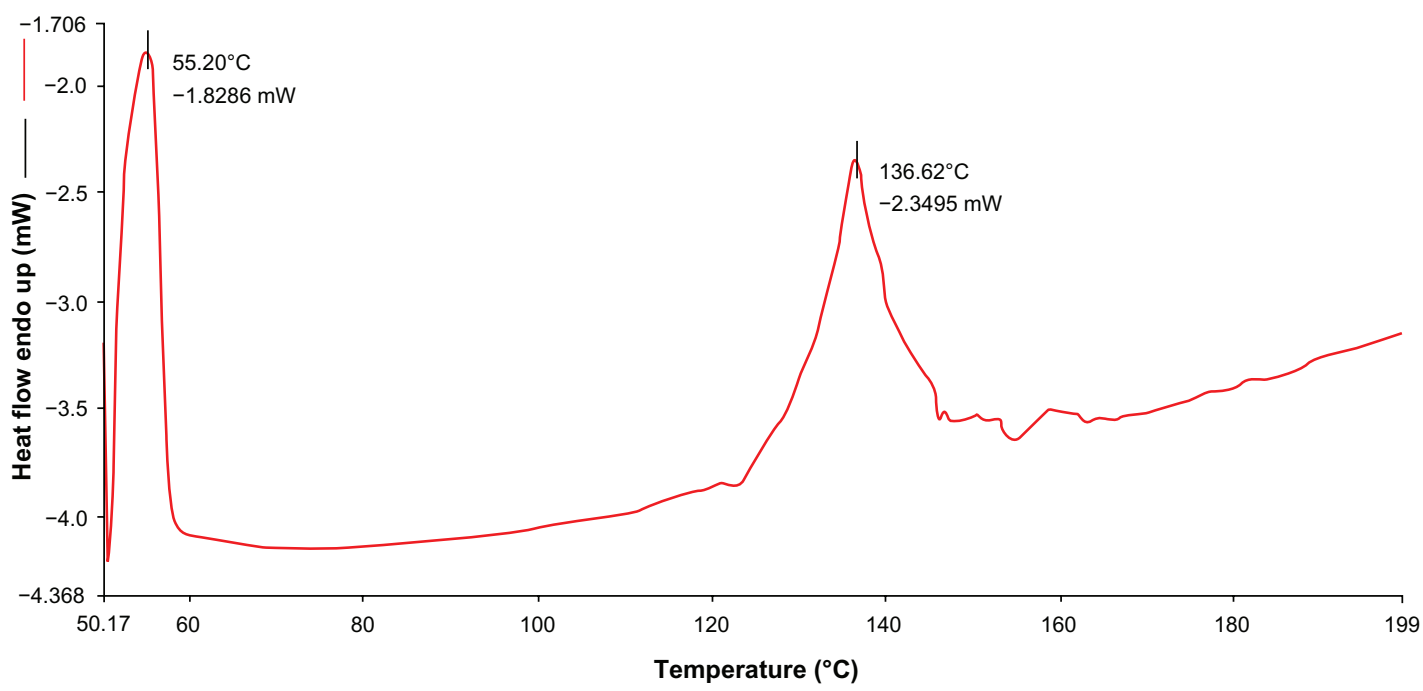

D

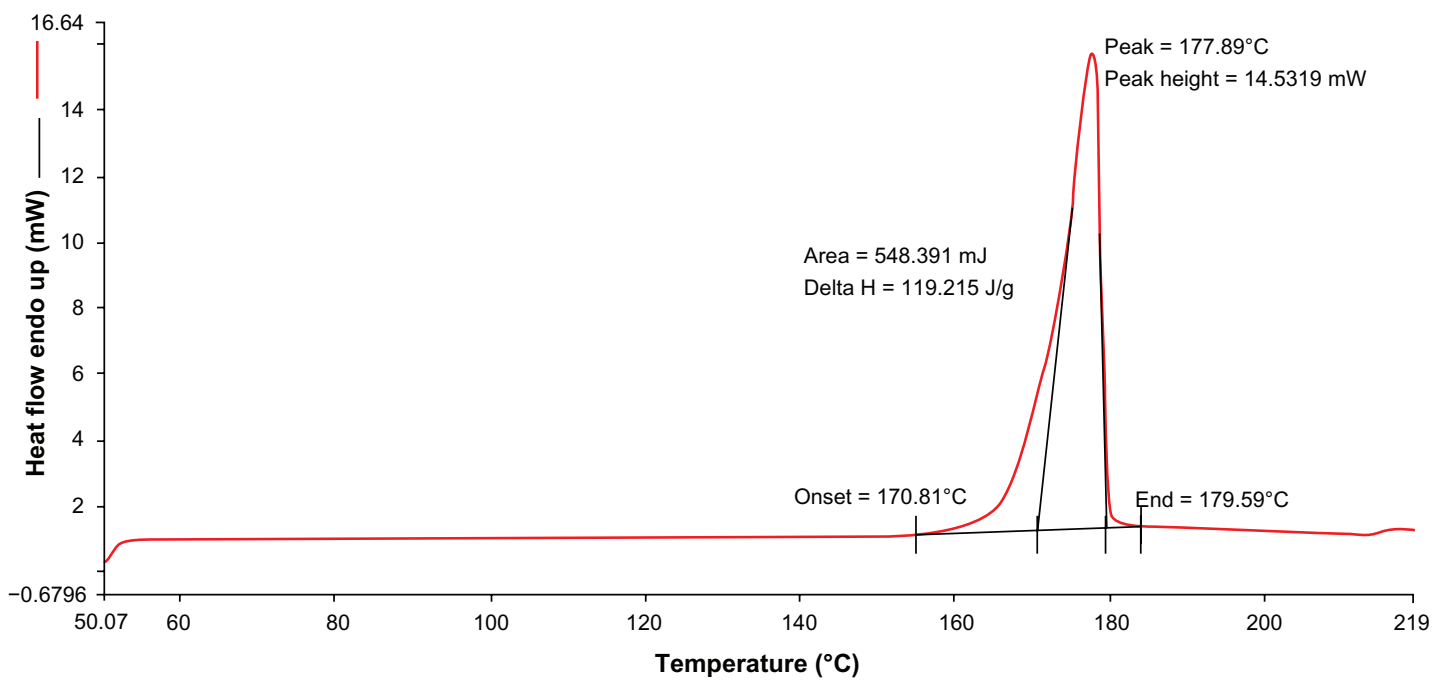

Figure 3 Differential scanning calorimetry (DSC) of blank NLCs (A), CUR-NLCs (B), physical mixture of blank NLCs and curcumin (C), and curcumin (D). Abbreviation: NLCs, nanostructured lipid carriers.

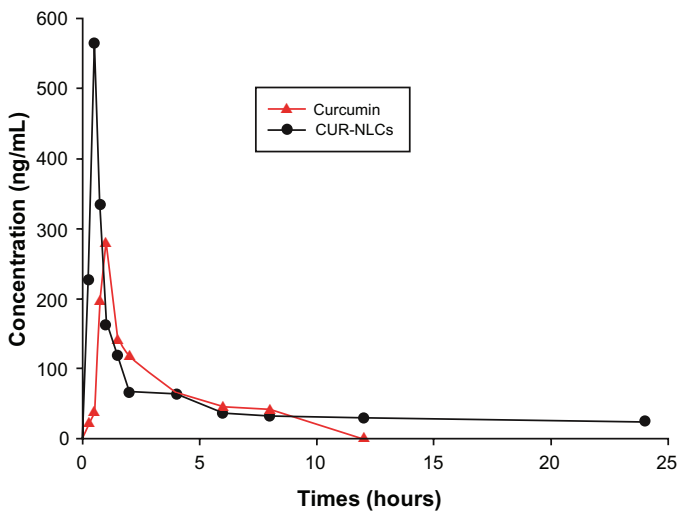

Figure 4 Plasma concentration time profiles of curcumin and CUR-NLCs suspension after intragastric administration in rats.

Notes: Data are mean \pm standard deviation, $\mathrm{n}=3$.

Abbreviation: CUR-NLCs, curcumin nanostructured lipid carriers.
Table 2 Pharmacokinetic parameters in rats after intragastric administration of CUR and CUR-NLCs $(n=5)$

\begin{tabular}{|c|c|c|}
\hline Parameters & CUR & CUR-NLCs \\
\hline$\alpha / h^{-1}$ & $0.58 \pm 0.11$ & $0.90 \pm 0.14$ \\
\hline$\beta / h^{-1}$ & $0.11 \pm 0.02$ & $0.03 \pm 0.0 \mathrm{I}^{\mathrm{b}}$ \\
\hline $\mathrm{Vc}, \mathrm{L} \cdot \mathrm{g}^{-1}$ & $0.83 \pm 0.12$ & $0.40 \pm 0.10$ \\
\hline $\mathrm{t}_{1 / 2} \alpha$, hours & $1.19 \pm 0.27$ & $0.77 \pm 0.13$ \\
\hline $\mathrm{t}_{\mathrm{I} / 2} \beta$, hours & $6.47 \pm 0.95$ & $20.62 \pm 1.91^{b}$ \\
\hline $\mathrm{AUC}_{0-\infty}, \mathrm{mg} \cdot \mathrm{h} \cdot \mathrm{L}^{-1}$ & $344.11 \pm 10.01$ & $820.36 \pm 25.11$ \\
\hline$C L(s), L \cdot g^{-1} \cdot h^{-1}$ & $0.23 \pm 0.07$ & $0.10 \pm 0.0 \mathrm{I}^{\mathrm{a}}$ \\
\hline MRT, hours & $2.94 \pm 0.82$ & $5.69 \pm 0.9 \mathrm{I}^{\mathrm{a}}$ \\
\hline
\end{tabular}

Notes: ${ }^{a} P<0.05$ vs CUR; ${ }^{b} P<0.01$ vs curcumin.

Abbreviations: CUR, curcumin suspension intragastric administration; CURNLCs, curcumin nanostructured lipid carriers intragastric administration; AUC, area under concentration curve; $\mathrm{CL}$, total plasma clearance; MRT, mean residence time; $t_{1 / 2} \alpha$, distribution half-life; $t_{1 / 2} \beta$, elimination half-life. 
the drug is released at its site of absorption. Therefore, the enhanced bioavailability of the CUR-NLC formulation might be attributable to uptake of nanoparticles via the gastrointestinal tract, increased permeability because of surfactants, and decreased degradation and clearance. ${ }^{31}$
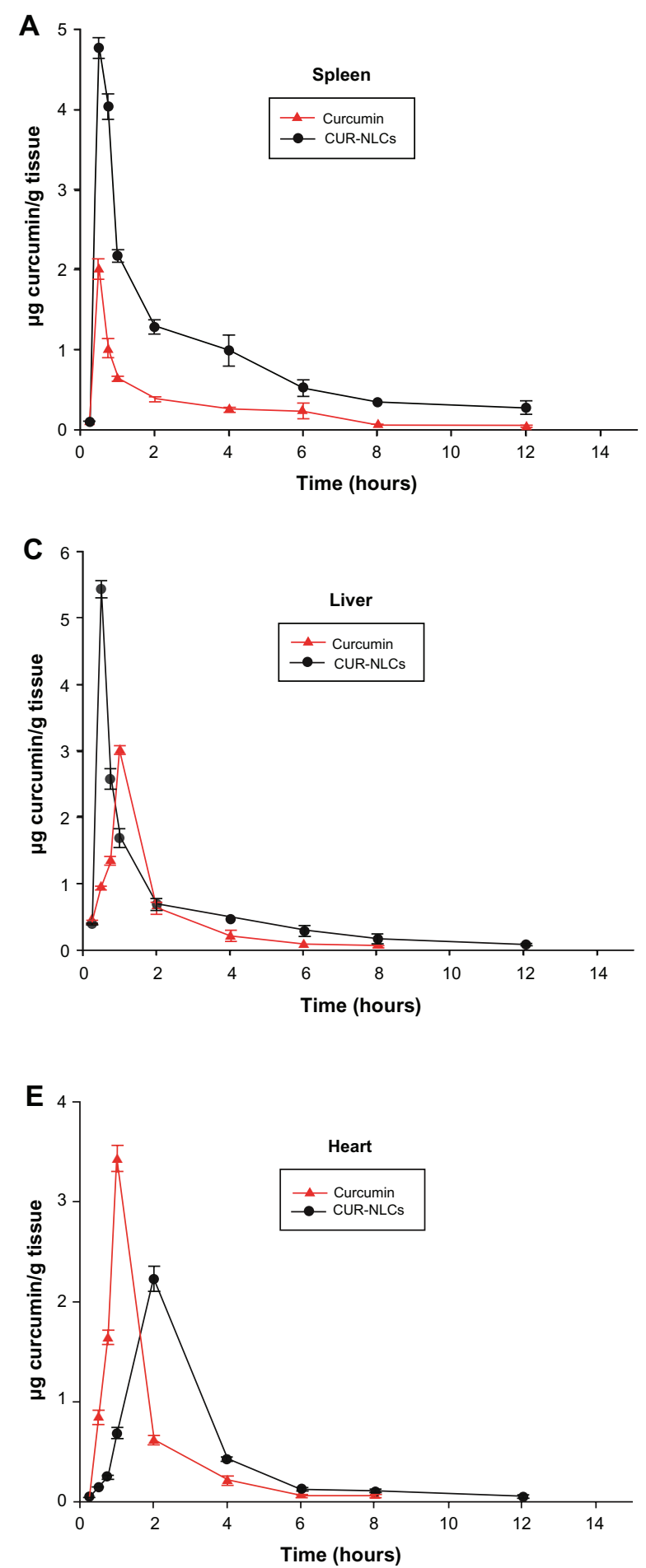

\section{Tissue distribution study}

An analytical method for measuring curcumin levels in tissue homogenates (liver, heart, spleen, lung, kidney, brain) has been reported previously. ${ }^{29}$ The limit of quantification in tissue for curcumin measurement was $50 \mathrm{ng} / \mathrm{mL}$. The calibration
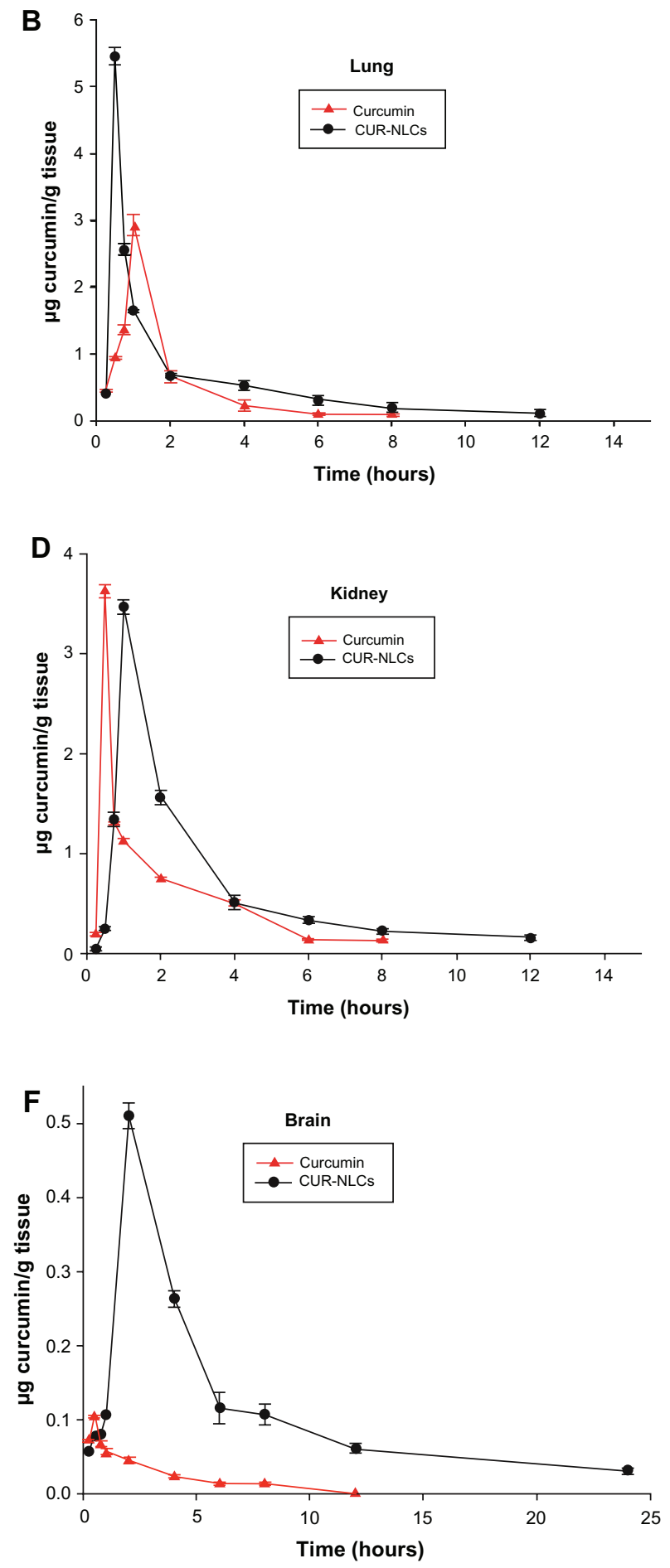

Figure 5 Tissue distribution curves of curcumin and CUR-NLCs after $80 \mathrm{mg}$ curcumin/kg intragastric administration in rats. Notes: Data are mean \pm standard deviation, $\mathrm{n}=3$.

Abbreviation: CUR-NLCs, curcumin nanostructured lipid carriers. 
curve over the range of $0.05-3.2 \mu \mathrm{g} / \mathrm{mL}$ for tissue homogenate had good linearity ( $\mathrm{R}^{2}$ was $\left.0.9994-0.9998\right)$. Intraday and interday accuracy precision was $2.7 \%-4.1 \%$ and $3.3 \%-4.7 \%$, respectively. The recovery of curcumin in the tissue homogenates was $96.4 \%-98.6 \%$.

The mean concentration-time curve (AUC) and corresponding pharmacokinetic parameters for curcumin and CUR-NLCs in rat organs for the two formulations are shown in Figure 5 and Table 3. After intragastric administration, curcumin and CUR-NLCs were distributed widely and could be detected in the heart, liver, spleen, lung, kidney, and brain tissue. As shown in Figure 5, in rats treated with the CUR-NLC solution, the peak plasma concentration in all tissues collected, except for the heart and brain, occurred at 30 minutes to 1 hour after treatment, and the peak plasma concentration occurred at 2 hours in the heart and brain. The peak plasma concentration in each specimen of rat tissue was increased using the nanoformulation, although this did not occur in kidney and heart tissue. In the present study, the peak plasma concentrations in the brain, liver, spleen, lung, heart, and kidney after administration of CUR-NLCs and curcumin suspension were 4.91, 2.32, 2.41, 1.80, 0.62 , and 0.96, respectively. After administration of the curcumin suspension, the $\mathrm{AUC}_{0-\infty}$ values were ranked as follows: heart $>$ kidney $>$ spleen $>$ lung $>$ liver $>$ brain.

Table 3 Pharmacokinetic parameters of curcumin and CURNLCs in rat organs following intragastric administration

\begin{tabular}{llll}
\hline Organs & $\mathbf{C}_{\max }(\mu \mathrm{g} / \mathbf{g})$ & AUC $(\mathbf{h} \mu \mathrm{g} / \mathbf{g})$ & MRT (h) \\
\hline Liver & & & \\
$\quad$ CUR & $1.98 \pm 0.04$ & $1.83 \pm 0.05$ & $1.86 \pm 0.02$ \\
$\quad$ CUR-NLCs & $4.59 \pm 0.10^{\mathrm{a}}$ & $3.22 \pm 0.04^{\mathrm{a}}$ & $2.12 \pm 0.01$ \\
Heart & & & \\
$\quad$ CUR & $3.50 \pm 0.19^{\mathrm{a}}$ & $4.77 \pm 0.02$ & $1.98 \pm 0.12$ \\
$\quad$ CUR-NLCs & $2.16 \pm 0.13$ & $5.14 \pm 0.13$ & $3.43 \pm 0.01^{\mathrm{a}}$ \\
Spleen & & & \\
$\quad$ CUR & $1.94 \pm 0.26$ & $3.44 \pm 0.09$ & $3.56 \pm 0.04$ \\
$\quad$ CUR-NLCs & $4.70 \pm 0.14$ & $10.73 \pm 0.17^{\mathrm{a}}$ & $5.10 \pm 0.08^{\mathrm{a}}$ \\
Lung & & & \\
$\quad$ CUR & $2.99 \pm 0.01$ & $3.04 \pm 0.02$ & $2.12 \pm 0.11$ \\
$\quad$ CUR-NLCs & $5.39 \pm 0.17^{\mathrm{a}}$ & $7.04 \pm 0.11^{\mathrm{a}}$ & $5.11 \pm 0.07^{\mathrm{a}}$ \\
Kidney & & & \\
CUR & $3.59 \pm 0.10$ & $4.10 \pm 0.19$ & $9.84 \pm 0.19$ \\
$\quad$ CUR-NLCs & $5.69 \pm 0.01$ & $5.16 \pm 0.26$ & $9.97 \pm 0.26$ \\
Brain & & & \\
$\quad$ CUR & $0.11 \pm 0.00$ & $0.25 \pm 0.01$ & $2.77 \pm 0.15$ \\
CUR-NLCs & $0.51 \pm 0.06^{\mathrm{b}}$ & $3.03 \pm 0.04^{\mathrm{b}}$ & $9.56 \pm 0.32^{\mathrm{b}}$ \\
\hline
\end{tabular}

Notes: ${ }^{a} P<0.05$ vs CUR; ${ }^{b} P<0.01$ vs curcumin.

Abbreviations: CUR, curcumin suspension intragastric administration; CURNLCs, curcumin nanostructured lipid carriers intragastric administration; $C_{\max }$, maximum tissue concentration of curcumin; AUC, area under concentration curve; MRT, mean residence time.
However, after administration of CUR-NLCs, the $\mathrm{AUC}_{0-\infty}$ values were ranked as spleen $>$ lung $>$ kidney $>$ heart $>$ liver $>$ brain. The AUC values in the brain, liver, heart, spleen, kidney, and lung of the two formulations were 11.93, $1.75,1.08,3.12,1.26$, and 2.32, respectively. From the increased AUC and mean residence time of curcumin, we conclude that our nanoformulation can increase curcumin levels significantly in these organs because of the increased AUC and mean residence time of curcumin. This indicates that CUR-NLCs may be more efficiently distributed to the organs than curcumin alone, and this might be attributed to uptake of the nanoparticles from the gastrointestinal tract and translocation to organs. The extent of biodistribution of particulate drug carriers is greatly influenced by their size, surface characteristics, and opsonization processes. It has been reported that opsonins can get adsorbed onto the nanoparticle surface and promote particle recognition by organs of the reticuloendothelial system, including the spleen, lung, liver, and kidney. ${ }^{26}$

High curcumin concentrations in various tissues are essential for curcumin to have pharmacological activity. It is noteworthy in this study that the $\mathrm{AUC}_{0-\infty}$ in the brain was 11.93. In recent studies, curcumin has shown substantial neuroprotective ability due to its antioxidant properties. It is well known that increasing the concentration of curcumin in the brain favors a protective effect in diseases of the brain. Curcumin was measurable in our brain homogenates for up to 24 hours after dosing in the CUR-NLC group, while the concentration was negligible in the curcumin suspension group. This indicates that CUR-NLCs can easily pass through the blood-brain barrier into brain tissue, which is essential for its neuroprotective effects.

According to our tissue distribution results, CUR-NLCs are rapidly absorbed, as evidenced by the shorter time taken to reach peak plasma concentration for the CUR-NLC formulation than for curcumin suspension. As a nanoformulation, the concentration and retention time for curcumin in the organs studied was significantly increased. Of all six tissues collected, the ratios of peak plasma concentration and $\mathrm{AUC}_{0-\infty}$ of the two formulations were the largest in the brain. For these reasons, the CUR-NLC formulation might be more effective than conventional curcumin for treating certain diseases of the brain. Further research is being carried out to investigate this.

\section{Conclusion}

In this study, curcumin, a poorly water-soluble drug, was incorporated in a nanostructured lipid carrier by the ethanol 
dripping method. Our research has shown that nanostructured lipid carriers can be exploited in order to enhance the incorporation of a poorly soluble drug. We demonstrated that curcumin and CUR-NLCs were distributed to the brain, heart, spleen, lung, liver, and kidney following intragastric administration. CUR-NLCs significantly raised the AUC and mean residence time of curcumin in all these organs, especially in the brain. Our results suggest that nanostructured lipid carriers are a promising delivery system for enhancing the oral absorption of poorly water-soluble drugs, including curcumin.

\section{Acknowledgments}

This study was supported by a grant from the National Natural Science Foundation of China (NSFC 81072291) and the National High Technology Research and Development Program of China (2010AA023003). We wish to thank Jason W Ashley from the Cell, Developmental, and Integrative Biology Department, University of Alabama at Birmingham, for his helpful suggestions.

\section{Disclosure}

The authors report no conflicts of interest in this work.

\section{References}

1. Sharma OP. Antioxidant activity of curcumin and related compounds. Biochem Pharmacol. 1976;25(15):1811-1812.

2. Selvam R, Subramanian L, Gayathri R, Angayarkanni N. The antioxidant activity of turmeric (Curcuma longa). $J$ Ethnopharmacol. 1995;47(2):59-67.

3. Ruby AJ, Kuttan G, Babu KD, Rajasekharan KN, Kuttan R. Antitumour and antioxidant activity of natural curcuminoids. Cancer Lett. 1995;94(1):79-83.

4. Menon VP, Sudheer AR. Antioxidant and anti-inflammatory properties of curcumin. Adv Exp Med Biol. 2007;595:105-125.

5. Rao TS, Basu N, Siddiqui HH. Anti-inflammatory activity of curcumin analogues. Indian J Med Res. 1982;75:574-578.

6. Negi PS, Jayaprakasha GK, Jagan Mohan Rao L, Sakariah KK. Antibacterial activity of turmeric oil: a byproduct from curcumin manufacture. J Agric Food Chem. 1999;47(10):4297-4300.

7. Wuthi-udomlert M, Grisanapan W, Luanratana O, Caichompoo W. Antifungal activity of Curcuma longa grown in Thailand. Southeast Asian J Trop Med Public Health. 2000;31(1):178-182.

8. Apisariyakul A, Vanittanakom N, Buddhasukh D. Antifungal activity of turmeric oil extracted from Curcuma longa (Zingiberaceae). J Ethnopharmacol. 1995;49(3):163-169.

9. Sharma M, Manoharlal R, Puri N, Prasad R. Antifungal curcumin induces reactive oxygen species and triggers an early apoptosis but prevents hyphae development by targeting the global repressor TUP1 in Candida albicans. Biosci Rep. 2010;30(6):391-404.

10. Lopez-Lazaro M. Anticancer and carcinogenic properties of curcumin: considerations for its clinical development as a cancer chemopreventive and chemotherapeutic agent. Mol Nutr Food Res. 2008;52 Suppl 1: S103-S127.

11. Das L, Vinayak M. Anti-carcinogenic action of curcumin by activation of antioxidant defence system and inhibition of NF-kappaB signalling in lymphoma-bearing mice. Biosci Rep. 2012;32(2):161-170.
12. Devasena T, Rajasekaran KN, Gunasekaran G, Viswanathan P, Menon VP. Anticarcinogenic effect of bis-1,7-(2-hydroxyphenyl)hepta-1,6-diene-3,5-dione a curcumin analog on DMH-induced colon cancer model. Pharmacol Res. 2003;47(2):133-140.

13. Park J, Conteas CN. Anti-carcinogenic properties of curcumin on colorectal cancer. World J Gastrointest Oncol. 2010;2(4):169-176.

14. Manikandan R, Thiagarajan R, Beulaja S, et al. Anti-cataractogenic effect of curcumin and aminoguanidine against selenium-induced oxidative stress in the eye lens of Wistar rat pups: An in vitro study using isolated lens. Chem Biol Interact. 2009;181(2):202-209.

15. Song JX, Sze SC, Ng TB, et al. Anti-Parkinsonian drug discovery from herbal medicines: what have we got from neurotoxic models? J Ethnopharmacol. 2012;139(3):698-711.

16. Miriyala S, Panchatcharam M, Rengarajulu P. Cardioprotective effects of curcumin. Adv Exp Med Biol. 2007;595:359-377.

17. Thiyagarajan M, Sharma SS. Neuroprotective effect of curcumin in middle cerebral artery occlusion induced focal cerebral ischemia in rats. Life Sci. 2004;74(8):969-985.

18. Shankar TN, Shantha NV, Ramesh HP, Murthy IA, Murthy VS. Toxicity studies on turmeric (Curcuma longa): acute toxicity studies in rats, guineapigs and monkeys. Indian J Exp Biol. 1980;18(1):73-75.

19. Lao CD, Ruffin MT 4th, Normolle D, et al. Dose escalation of a curcuminoid formulation. BMC Complement Altern Med. 2006;6:10.

20. Anand P, Kunnumakkara AB, Newman RA, Aggarwal BB. Bioavailability of curcumin: problems and promises. Mol Pharm. 2007;4(6):807-818.

21. Pan MH, Huang TM, Lin JK. Biotransformation of curcumin through reduction and glucuronidation in mice. Drug Metab Dispos. 1999;27(4):486-494.

22. Sharma RA, Steward WP, Gescher AJ. Pharmacokinetics and pharmacodynamics of curcumin. Adv Exp Med Biol. 2007;595:453-470.

23. Luo CF, Yuan M, Chen MS, et al. Pharmacokinetics, tissue distribution and relative bioavailability of puerarin solid lipid nanoparticles following oral administration. Int J Pharm. 2011;410(1/2):138-144.

24. Tiyaboonchai W, Tungpradit W, Plianbangchang P. Formulation and characterization of curcuminoids loaded solid lipid nanoparticles. Int J Pharm. 2007;337(1/2):299-306.

25. Gomez-Gaete C, Fattal E, Silva L, Besnard M, Tsapis N. Dexamethasone acetate encapsulation into Trojan particles. J Control Release. 2008;128(1):41-49.

26. Yang BC, Chu ZF, Zhu S, et al. Study of pharmacokinetics and tissue distribution of liposomal brucine for dermal administration. Int $J$ Nanomedicine. 2011;6:1109-1116.

27. Wang MT, Jin Y, Yang YX, et al. In vivo biodistribution, antiinflammatory, and hepatoprotective effects of liver targeting dexamethasone acetate loaded nanostructured lipid carrier system. Int $J$ Nanomedicine. 2010;5:487-497.

28. Mu L, Feng SS. Fabrication, characterization and in vitro release of paclitaxel (Taxol) loaded poly (lactic-co-glycolic acid) microspheres prepared by spray drying technique with lipid/cholesterol emulsifiers. J Control Release. 2001;76(3):239-254.

29. Tsai YM, Chien CF, Lin LC, Tsai TH. Curcumin and its nano-formulation: the kinetics of tissue distribution and blood-brain barrier penetration. Int J Pharm. 2011;416(1):331-338.

30. Paliwal R, Rai S, Vaidya B, et al. Effect of lipid core material on characteristics of solid lipid nanoparticles designed for oral lymphatic delivery. Nanomedicine. 2009;5(2):184-191.

31. Tiwari R, Pathak K. Nanostructured lipid carrier versus solid lipid nanoparticles of simvastatin: comparative analysis of characteristics, pharmacokinetics and tissue uptake. Int J Pharm. 2011;415(1/2):232-243.

32. des Rieux A, Fievez V, Momtaz M, et al. Helodermin-loaded nanoparticles: characterization and transport across an in vitro model of the follicleassociated epithelium. J Control Release. 2007;118(3):294-302.

33. Sahu A, Bora U, Kasoju N, Goswami P. Synthesis of novel biodegradable and self-assembling methoxy poly(ethylene glycol)-palmitate nanocarrier for curcumin delivery to cancer cells. Acta Biomater. 2008;4(6):1752-1761. 


\section{Publish your work in this journal}

The International Journal of Nanomedicine is an international, peerreviewed journal focusing on the application of nanotechnology in diagnostics, therapeutics, and drug delivery systems throughout the biomedical field. This journal is indexed on PubMed Central, MedLine, CAS, SciSearch $\AA$, Current Contents ${ }^{\circledR} /$ Clinical Medicine,

Journal Citation Reports/Science Edition, EMBase, Scopus and the Elsevier Bibliographic databases. The manuscript management system is completely online and includes a very quick and fair peer-review system, which is all easy to use. Visit http://www.dovepress.com/ testimonials.php to read real quotes from published authors.

Submit your manuscript here: http://www.dovepress.com/international-journal-of-nanomedicine-journal 